\title{
Superconducting spin valves based on epitaxial Fe/V superlattices
}

\author{
G. Nowak, ${ }^{*}$ H. Zabel, and K. Westerholt \\ Experimentalphysik/Festkörperphysik, Ruhr-Universität Bochum, D-44780 Bochum, Germany
}

I. Garifullin

Zavoisky Physical-Technical Institute, Kazan Scientific Center of Russian Academy of Sciences, 420029 Kazan, Russia

\author{
M. Marcellini, A. Liebig, and B. Hjörvarsson \\ Department of Physics, Uppsala University, Box 530, 75121 Uppsala, Sweden
}

(Received 14 July 2008; revised manuscript received 2 September 2008; published 15 October 2008)

\begin{abstract}
In superconducting spin valves of the type $\mathrm{S} / \mathrm{F} 1 / \mathrm{N} / \mathrm{F} 2$ or $\mathrm{F} 1 / \mathrm{S} / \mathrm{F} 2$ with a superconducting layer $\mathrm{S}$, two ferromagnetic layers $\mathrm{F} 1$ and $\mathrm{F} 2$, and a normal metallic layer $\mathrm{N}$, the superconducting transition temperature $T_{\mathrm{S}}$ depends on the relative magnetization direction of the ferromagnetic layers $F 1$ and F2. The difference of the transition temperature $\Delta T_{\mathrm{S}}=T_{s}^{\mathrm{AP}}-T_{s}^{\mathrm{P}}$ with the magnetization direction of $\mathrm{F} 1$ and F2 either antiparallel or parallel is called the superconducting spin valve effect. We have prepared both types of spin valves by growing $\mathrm{Fe} / \mathrm{V}$ thin-film heterostructures with epitaxial quality on $\mathrm{MgO}(001)$ substrates. In the S/F1/N/F2-type spin valves the ferromagnetic layers were the first two Fe layers of a $[\mathrm{Fe} / \mathrm{V}]$ superlattice coupled antiferromagnetically via the interlayer exchange interaction. Here we observed a superconducting spin valve shift of up to $\Delta T_{\mathrm{S}} \approx 200 \mathrm{mK}$ when aligning the sublattice magnetization in an external magnetic field. In the F1/S/F2-type spin valves the ferromagnetic layer $\mathrm{F} 1$ was either a $[\mathrm{Fe} / \mathrm{V}]$ or a $\left[\mathrm{Fe}_{x} \mathrm{~V}_{1-x} / \mathrm{V}\right]$ superlattice, the $\mathrm{F} 2$ layer was a $\mathrm{Fe}-$, a Co-, or a $\mathrm{Fe}_{x} \mathrm{~V}_{1-x}$ film. Using weakly ferromagnetic $\mathrm{Fe}_{x} \mathrm{~V}_{1-x}$ alloy layers as $\mathrm{F} 1$ and $\mathrm{F} 2$ we find a spin valve effect of up to $\Delta T_{\mathrm{S}} \approx 20 \mathrm{mK}$, which is more than a factor of 2 larger than reported in the literature before for spin valves with comparable transition temperatures. Our results indicate that a high interface transparency and a large superconducting correlation length are prerequisites for the observation of a sizable superconducting spin valve effect.
\end{abstract}

DOI: 10.1103/PhysRevB.78.134520

PACS number(s): 74.45.+c, 74.78.-w, 73.50.-h, 75.70.-i

\section{INTRODUCTION}

The complex interplay between superconductivity and ferromagnetism in thin-film systems combined of superconducting (S) and ferromagnetic (F) layers is a topic of great current interest and characterized by spectacular theoretical predictions waiting for experimental realization (see Refs. 1-6 for recent reviews). Topics under intense experimental investigation at present are possible triplet superconductivity in $\mathrm{S} / \mathrm{F}$ systems and the inverse proximity effect, i.e., a penetration of itinerant ferromagnetism into the $\mathrm{S}$ layer from the F layer (see, e.g., Efetov et al. in Ref. 6). The physics of the proximity effect at the S/F interface is rich in phenomena and only quite recently the theoretical work has been extended to include strong ferromagnets such as $\mathrm{Fe}, \mathrm{Co}$, and Ni. Strong ferromagnets with a large exchange splitting between the spin-up and the spin-down electron bands favor triplet superconductivity. 5,7

An important consequence of the $\mathrm{S} / \mathrm{F}$ proximity effect is the occurrence of $\pi$-wave superconductivity in S/F/S Josephson junctions. The propagating character of the pair wave function in the F layer causes a phase shift of $\pi$ between the two superconductors for a certain thickness range of the $\mathrm{F}$ layer. $^{8-11} \pi$-wave superconductivity in S/F/S Josephson junctions is firmly established in the literature now and already applied in devices for the realization of superconducting qubits for quantum computing. ${ }^{10,12}$

The theory of another interesting device based on the S/F proximity effect, the superconducting spin valve, has been developed about ten years ago by Tagirov ${ }^{13}$ and Buzdin et $a l .{ }^{14}$ In superconducting spin valves with the layer sequence F1/S/F2 the superconducting transition temperature $T_{\mathrm{S}}$ of the $\mathrm{S}$ layer can be controlled by the relative orientation of the magnetization direction of the two ferromagnetic layers F1 and F2. Model calculations show that the transition temperature with antiparallel orientation of $\mathrm{F} 1$ and $\mathrm{F} 2 T_{\mathrm{S}}^{\mathrm{AP}}$ should be larger than the transition temperature $T_{\mathrm{S}}^{\mathrm{P}}$ for a parallel orientation of F1 and F2. Thus one can principally switch the superconductivity on and off by reversing the magnetization direction of F1 and F2, corresponding to an infinite magnetoresistance. Needless to say that a working device of this type would be highly desirable in the emerging field of superconducting spintronics. ${ }^{8-12}$ The basic physical reason for the difference in the superconducting transition temperature $\Delta T_{\mathrm{S}}=T_{\mathrm{S}}^{\mathrm{AP}}-T_{\mathrm{S}}^{\mathrm{P}}$ is the partial compensation of the pair breaking ferromagnetic exchange field, if the magnetization directions of F1 and F2 are aligned antiparallel.

The first experimental confirmation of the superconducting spin valve effect was reported by $\mathrm{Gu}$ et al. ${ }^{15}$ for a $\mathrm{NiCu} /$ $\mathrm{Nb} / \mathrm{NiCu}$ trilayer structure, however, with a small difference $\Delta T_{\mathrm{S}}=T_{\mathrm{S}}^{\mathrm{AP}}-T_{\mathrm{S}}^{\mathrm{P}}=6 \mathrm{mK}$ only. Successively several groups published results on superconducting spin valves of different design. ${ }^{16-19}$ The maximum shift $\Delta T_{\mathrm{S}} \approx 40 \mathrm{mK}$ was reported by Moraru et al. ${ }^{17}$ for a $\mathrm{Ni} / \mathrm{Nb} / \mathrm{Ni}$ trilayer, however, for a sample with a very low superconducting transition temperature of $T_{\mathrm{S}}=0.4 \mathrm{~K}$ and with a resistive transition width of about $0.3 \mathrm{~K}$. For thicker $\mathrm{Nb}$ layers in $\mathrm{Ni} / \mathrm{Nb} / \mathrm{Ni}$ with transition temperatures in the range of $2 \mathrm{~K} \Delta T_{\mathrm{S}}$ drops again below $10 \mathrm{mK}$ (Ref. 17). At variance to these results also small negative shifts $\Delta T_{\mathrm{S}}=T_{\mathrm{S}}^{\mathrm{AP}}-T_{\mathrm{S}}^{\mathrm{P}}$ in $\mathrm{F} 1 / \mathrm{S} / \mathrm{F} 2$ systems have been 
reported. ${ }^{18,19}$ Rusanov et al. in Ref. 18 found $\Delta T_{\mathrm{S}}=-2 \mathrm{mK}$ for a $\mathrm{FeCo} / \mathrm{Nb} / \mathrm{FeCo}$ trilayer, Singh et al. ${ }^{19}$ for $\mathrm{CoPt} / \mathrm{Nb} /$ $\mathrm{CoPt}$, a system with an easy magnetic axis perpendicular to the film plane, observed a shift of $\Delta T_{\mathrm{S}}=-5 \mathrm{mK}$. This negative shift, dubbed as the "inverse superconducting spin valve effect" in Ref. 18 is thought to be caused by spin accumulation in the layers, which depends on the relative spin orientation of F1 and F2. From these results doubts arose on the existence of the superconducting spin valve effect in systems with strongly ferromagnetic layers.

Since in any case the observed shifts $\Delta T_{\mathrm{S}}$ due to the superconducting spin valve effect or the inverse superconducting spin valve effect are small, a possible influence of magnetic stray fields from magnetic domains of the ferromagnetic layers $\mathrm{F} 1$ and $\mathrm{F} 2$ on the resistive transition must carefully be considered. This has clearly been demonstrated by Steiner and Ziemann, ${ }^{20}$ who showed that in $\mathrm{Fe} /$ $\mathrm{Nb} / \mathrm{Co}$ trilayers and in $\mathrm{Co} / \mathrm{Nb}$ bilayers the stray fields in the multidomain state of the ferromagnet can appreciably change the superconducting transition temperature measured resistively. Actually, in a trilayer with an essentially antiparallel orientation of F1 and F2, the residual stray fields can shift $T_{\mathrm{S}}^{\mathrm{AP}}$ below $T_{\mathrm{S}}^{\mathrm{P}}$, thus mimicking a negative value for the spin valve shift $\Delta T_{\mathrm{S}} \cdot{ }^{20}$ The main reason for this is that in the narrow magnetic-field range between the coercive fields of F1 and F2, where the antiparallel orientation is established, magnetic domains may still exist; i.e., the antiparallel orientation of F1 and F2 might not be perfect.

In contrast to the experimental results, theoretical model calculations ${ }^{13,14}$ with an optimum set of microscopic superconducting parameters predict a superconducting spin valve shift $\Delta T_{\mathrm{S}}$ of the order of several $K$. The microscopic origin of this discrepancy is not very clear yet, but a detailed inspection of the model calculations gives important hints. A parameter of primary importance for the superconducting spin valve effect is the ratio $\xi_{\mathrm{S}} / d_{\mathrm{S}}$ with the coherence length $\xi_{\mathrm{S}}$ and thickness $d_{\mathrm{S}}$ of the superconducting layer. Large shifts of the superconducting transition temperatures are only expected if $\xi_{\mathrm{S}} / d_{\mathrm{S}} \geq 1$. The physical reason for this is that a Cooper pair should simultaneously sense the ferromagnetic exchange field of both ferromagnetic layers F1 and F2. For the opposite case $\xi_{\mathrm{S}} / d_{\mathrm{S}} \leq 1$ the shift of the superconducting transition temperature $\Delta T_{\mathrm{S}}$ vanishes exponentially with increasing superconducting film thickness. ${ }^{13,14}$ In all experimental systems studied so far ${ }^{15,16,18,19} \xi_{\mathrm{S}} / d_{\mathrm{S}} \leq 0.4$ holds, and for this case also the model calculations predict only a small superconducting spin valve effect.

A parameter of similar importance for the spin valve effect is the quantum-mechanical transparency of the $S / F$ interface. Due to electron band mismatch for at least one of the spin-up or spin-down subbands in typical S/F material combinations, the transparency of the $\mathrm{S} / \mathrm{F}$ interfaces for Cooper pairs is usually low (see, e.g., Ref. 6 for a discussion). A small transparency parameter $T$ (see Ref. 13 for the exact definition) severely limits the superconducting spin valve effect. ${ }^{13}$ A possible method to increase the interface transparency is the dilution of the ferromagnet. With decreasing ferromagnetic exchange splitting between the spin-up and spindown electron band the band mismatch becomes less pronounced and the transparency can increase correspond- ingly, as was demonstrated for the $\mathrm{V} / \mathrm{Fe}_{x} \mathrm{~V}_{1-x}$ interface by Aarts et al. ${ }^{21}$ In addition, strong spin-orbit scattering of the conduction electrons in the $\mathrm{S}$ layer or paramagnetic pair breaking scattering at magnetic impurities at the $\mathrm{S} / \mathrm{F}$ interfaces can be very detrimental for the superconducting spin valve effect. ${ }^{13,14}$

Thus a careful selection of a suitable $\mathrm{S} / \mathrm{F}$ system is a critical starting point for a convincing demonstration of the spin valve effect. Since the quality of the S/F interface is of utmost importance, a fully epitaxial $\mathrm{S} / \mathrm{F}$ layer system with high quality interfaces should be the best choice. As will be detailed below, in our opinion the $\mathrm{V} / \mathrm{Fe}$ interface or, even more so, the $\mathrm{V} / \mathrm{Fe}_{x} \mathrm{~V}_{1-x}$ interface is the best candidate fulfilling most of the important requirements. Bilayers, trilayers, and multilayers of Fe/V systems have contributed substantially to the S/F-proximity effect problem over the years, starting from the early work of Wong et al. (Ref. 22). Recently we have studied a superconducting $V$ film grown on an epitaxial $[\mathrm{Fe} / \mathrm{V}]$ superlattice with antiferromagnetic interlayer exchange coupling and we have shown that the superconducting transition temperature $T_{\mathrm{S}}$ depends on the relative orientation of the magnetization direction of successive $\mathrm{Fe}$ layers in the $[\mathrm{Fe} / \mathrm{V}]$ superlattice. ${ }^{23}$ Actually this is a variant of the superconducting spin valve effect discussed above, only that here the spin valve has both ferromagnetic layers $\mathrm{F} 1$ and $\mathrm{F} 2$ on the same side of the superconducting film (layer sequence is $\mathrm{S} / \mathrm{F} 1 / \mathrm{N} / \mathrm{F} 2$ ). The $\mathrm{S} / \mathrm{F} 1 / \mathrm{N} / \mathrm{F} 2$-type spin valve with a highly transparent normal metallic layer $\mathrm{N}$ decoupling the two ferromagnetic layers was treated theoretically by Oh et al. in Ref. 24. The main result is the same as discussed above, namely, that one expects a difference $\Delta T_{\mathrm{S}}$ in the superconducting transition temperature for the magnetization direction of F1 and F2 in the antiparallel orientation and in the parallel orientation. Remarkably, we observed a shift $\Delta T_{\mathrm{S}}=120 \mathrm{mK}$ for a $18 \mathrm{~nm}$ thick $V$ film on a $[\mathrm{Fe}(2 \mathrm{ML}) / \mathrm{V}(12 \mathrm{ML})]_{25}$ superlattice (ML: monolayer), ${ }^{23}$ which is nearly one order of magnitude larger than observed for the F1/S/F2-type spin valves up to now.

Based on this experience we prepared both types of spin valves by growing different $\mathrm{Fe} / \mathrm{V}$ thin-film structures with epitaxial quality on $\mathrm{MgO}(001)$ substrates. In continuation of our previous work we first study S/F1/N/F2-type spin valves for different thicknesses of the Fe layer in the superlattice. Then we prepared fully epitaxial F1/S/F2-type structures and investigated the spin valve effect for F1 and F2 layers with different composition, thickness, and quality.

The paper is organized as follows: we first analyze the superconducting properties of a single $V$ film deposited on a $[\mathrm{Fe}(\mathrm{nML}) / \mathrm{V}(\mathrm{mML})]$ superlattice by varying either the number of Fe monolayers $n$ or the number of $V$ monolayers $m$. Then, for selected combinations of $n$ and $m$, we deposit a second ferromagnetic layer F2, either Fe or Co, on top of the $V$ film. Finally, we replace the Fe layers in the superlattice and in the top layer by ferromagnetic $\mathrm{Fe}_{x} \mathrm{~V}_{1-x}$ alloy layers.

\section{PREPARATION AND EXPERIMENT}

The samples were prepared by dc-magnetron sputtering in an UHV system with a base pressure of $2 \times 10^{-10}$ mbar on 
TABLE I. Layer sequence of all samples used for the present study with the residual resistivity ratio RRR, the ratio $\xi_{\mathrm{S}} / d_{\mathrm{S}}$, the superconducting transition temperature $T_{s}$, and the shift $\Delta T_{s}$ (see main text).

\begin{tabular}{|c|c|c|c|c|c|}
\hline Series & Sample & RRR & $\xi_{\mathrm{S}} / d_{\mathrm{S}}$ & $\begin{array}{c}T_{s} \\
(\mathrm{~K})\end{array}$ & $\begin{array}{c}\Delta T_{s} \\
(\mathrm{mK})\end{array}$ \\
\hline 1 & $\begin{array}{c}\mathrm{Pd}(5 \mathrm{~nm}) / \mathrm{V}(24.2 \mathrm{~nm}) /[\mathrm{Fe}(\mathrm{nxML}) / \mathrm{V}(8 \mathrm{ML})]_{25} / \mathrm{MgO}(001) \\
n=1 \ldots 14\end{array}$ & $\begin{array}{c}11 \ldots 17 \\
\text { (see Fig. 4) }\end{array}$ & $0.5 \ldots 0.80$ & $\begin{array}{c}2 \ldots 3 \\
\text { (see Fig. 4) }\end{array}$ & \\
\hline 2 & $\begin{array}{c}\mathrm{Pd}(5 \mathrm{~nm}) / \mathrm{V}(24.2 \mathrm{~nm}) /[\mathrm{Fe}(3 \mathrm{ML}) / \mathrm{V}(\mathrm{mxML})]_{25} / \mathrm{MgO}(001) \\
m=8 \ldots 15\end{array}$ & $11 \ldots 16$ & $0.5 \ldots 0.78$ & $2 \ldots 3$ & \\
\hline 3 & $\begin{array}{c}\text { F1/S/F2-type SC spin valves } \\
\text { Co or Fe top layer }\end{array}$ & & & & \\
\hline a & $\begin{array}{l}\mathrm{CoO}(3 \mathrm{~nm}) / \mathrm{Co}(7 \mathrm{~nm}) / \mathrm{V}(24.5 \mathrm{~nm}) / \\
{[\mathrm{Fe}(3 \mathrm{ML}) / \mathrm{V}(8 \mathrm{ML})]_{15} / \mathrm{MgO}(001)}\end{array}$ & 11 & 0.55 & 1.97 & $4 \mp 1$ \\
\hline $\mathrm{b}$ & $\begin{array}{c}\mathrm{CoO}(3 \mathrm{~nm}) / \mathrm{Co}(7 \mathrm{~nm}) / \mathrm{Fe}(5 \mathrm{~nm}) / \mathrm{V}(35.2 \mathrm{~nm}) / \\
{[\mathrm{Fe}(3 \mathrm{ML}) / \mathrm{V}(8 \mathrm{ML})]_{10} / \mathrm{MgO}(001)}\end{array}$ & 10.5 & 0.37 & 2.03 & $3 \mp 1$ \\
\hline $\mathrm{c}$ & $\begin{array}{c}\mathrm{CoO}(3 \mathrm{~nm}) / \mathrm{Co}(7 \mathrm{~nm}) / \mathrm{Fe}(5 \mathrm{~nm}) / \mathrm{V}(30.3 \mathrm{~nm}) / \\
{[\mathrm{Fe}(3 \mathrm{ML}) / \mathrm{V}(8 \mathrm{ML})]_{10} / \mathrm{MgO}(001)}\end{array}$ & 9.5 & 0.43 & 1.42 & $6 \mp 2$ \\
\hline & $\mathrm{Fe}_{x} \mathrm{~V}_{1-x}$ alloy top layers & & & & \\
\hline d & $\begin{array}{c}\mathrm{CoO}(3 \mathrm{~nm}) / \mathrm{Co}(7 \mathrm{~nm}) / \mathrm{Fe}_{47} \mathrm{~V}_{53}(10 \mathrm{~nm}) / \mathrm{V}(37.8 \mathrm{~nm}) / \\
{[\mathrm{Fe}(3 \mathrm{ML}) / \mathrm{V}(8 \mathrm{ML})]_{10} / \mathrm{MgO}(001)}\end{array}$ & 13 & 0.42 & 2.27 & $4 \mp 2$ \\
\hline e & $\begin{array}{c}\mathrm{CoO}(3 \mathrm{~nm}) / \mathrm{Co}(7 \mathrm{~nm}) / \mathrm{Fe}_{32} \mathrm{~V}_{68}(3.7 \mathrm{~nm}) / \mathrm{V}(37.8 \mathrm{~nm}) / \\
{\left[\mathrm{Fe}_{32} \mathrm{~V}_{68}(15 \mathrm{ML}) / \mathrm{Fe}(3 \mathrm{ML})\right]_{8} / \mathrm{MgO}(001)}\end{array}$ & 13 & 0.40 & 2.84 & $7 \mp 2$ \\
\hline $\mathrm{f}$ & $\begin{array}{c}\mathrm{CoO}(3 \mathrm{~nm}) / \mathrm{Co}(7 \mathrm{~nm}) / \mathrm{Fe}_{32} \mathrm{~V}_{68}(10 \mathrm{~nm}) / \mathrm{V}(34.8 \mathrm{~nm}) / \\
{[\mathrm{Fe}(3 \mathrm{ML}) / \mathrm{V}(8 \mathrm{ML})]_{10} / \mathrm{MgO}(001)}\end{array}$ & 13 & 0.40 & 2.39 & $10 \mp 2$ \\
\hline $\mathrm{g}$ & $\begin{array}{c}\mathrm{CoO}(3 \mathrm{~nm}) / \mathrm{Co}(7 \mathrm{~nm}) / \mathrm{Fe}_{32} \mathrm{~V}_{68}(3.7 \mathrm{~nm}) / \mathrm{V}(31 \mathrm{~nm}) / \\
{\left[\mathrm{Fe}_{32} \mathrm{~V}_{68}(15 \mathrm{ML}) / \mathrm{V}(8 \mathrm{ML})\right]_{8} / \mathrm{MgO}(001)}\end{array}$ & 11 & 0.46 & 2.23 & $20 \mp 1$ \\
\hline $\mathrm{h}$ & $\begin{array}{c}\mathrm{CoO}(3 \mathrm{~nm}) / \mathrm{Co}(7 \mathrm{~nm}) / \mathrm{Fe}_{24} \mathrm{~V}_{76}(3.7 \mathrm{~nm}) / \mathrm{V}(24.2 \mathrm{~nm}) / \\
{\left[\mathrm{Fe}_{24} \mathrm{~V}_{76}(15 \mathrm{ML}) / \mathrm{Fe}(3 \mathrm{ML})\right]_{8} / \mathrm{MgO}(001)}\end{array}$ & 14.6 & 0.67 & 2.32 & $12 \mp 1$ \\
\hline
\end{tabular}

$\mathrm{MgO}(100)$ single crystalline substrates at a substrate temperature of $320^{\circ} \mathrm{C}$. For more details on the preparation procedure see Isberg et $a .^{25}$ In the first preparation step we deposited a $[\mathrm{Fe}(\mathrm{nML}) / \mathrm{V}(\mathrm{mML})]$ superlattice, starting with the $V$ layer and finishing with the Fe layer. Then the thick superconducting $V$ layer was deposited and either capped by a $5 \mathrm{~nm}$ thick $\mathrm{Pd}$ film as a protection against oxidation [samples of series 1 and series 2 (see Table I)] or covered by the second ferromagnetic layer F2, which was either Co, Fe, or $\mathrm{Fe}_{x} \mathrm{~V}_{1-x}$ [samples of series 3 (see Table I)]. In the latter case we deposited an additional thin Co layer on top of the F2 layer. About $3 \mathrm{~nm}$ of this layer oxidize in air forming $\mathrm{CoO}$, which serves as an exchange bias layer in order to increase the coercive field $H_{C}$ of the F2 layer. (For a review on exchange bias systems see the paper by Nogués and Shuller. ${ }^{26}$ ) Table I gives an overview of the layer sequence of all samples prepared for the present study.

The x-ray structural characterization was carried out using synchrotron radiation at the beam line W1.1 at HaSyLab on a six-circle diffractometer at the photon energy of $8048 \mathrm{eV}$, corresponding to the $\mathrm{Cu} K_{\alpha 1}$ wavelength of $\lambda=0.154 \mathrm{~nm}$. The primary slit was set to $0.2 \mathrm{~mm}$; the secondary slit fixed at $0.5 \mathrm{~mm}$ giving a beam divergence of $0.015^{\circ}$. The diffractometer enables a complete structural characterization of thin films, including small-angle x-ray reflectivity, Bragg scans, and in-plane and out-of-plane rocking scans.
The magnetic hysteresis loops were measured in a commercial superconducting quantum interference device (SQUID) magnetometer (Quantum Design MPMS system) at a temperature of $10 \mathrm{~K}$. The electrical resistivity measurements were performed in a standard four contact current-inplane (CIP) configuration with silver painted contacts using a $\mathrm{He}^{4}$ bath cryostat (Oxford) equipped with a pair of superconducting solenoids in Helmholtz geometry for the generation of a magnetic field up to $5 \mathrm{~T}$. The sample plane can be adjusted with a precision of $0.1^{\circ}$ parallel to the field axis by rotation of the sample rod, which is important for the precise determination of the upper critical magnetic field $\mathrm{H}_{c 2}^{p}(T)$ with the direction of the film plane parallel to the field. The temperature dependence of the upper critical field was also measured by resistance measurements in an applied field. During the observation of the superconducting transition the sample holder was immersed in liquid $\mathrm{He}$ and the liquid was slowly pumped off at a cooling rate of about $0.05 \mathrm{~K} / \mathrm{min}$. This allowed a reproducibility of the superconducting transition temperature with a precision of about $1 \mathrm{mK}$.

\section{RESULTS AND DISCUSSION}

\section{A. Structural properties}

In Fig. 1 we show small-angle reflectivity scans for three selected examples from the three series of samples in Table I. 


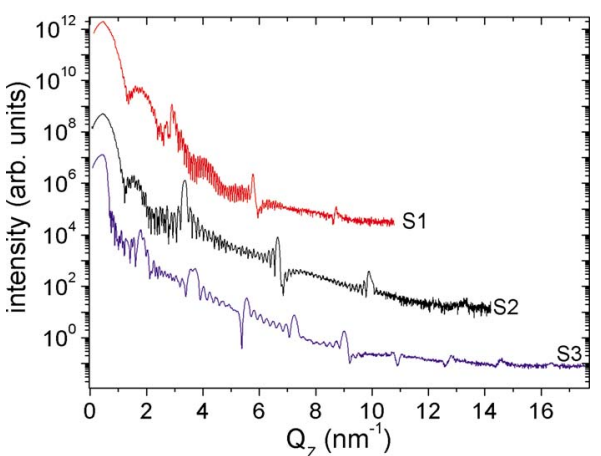

FIG. 1. (Color online) Small-angle x-ray reflectivity scans for selected samples from Table I. S1 $\mathrm{Pd}(5 \mathrm{~nm}) / \mathrm{V}(24.2 \mathrm{~nm}) /$ $[\mathrm{Fe}(5 \mathrm{ML}) / \mathrm{V}(8 \mathrm{ML})]_{25}, \mathrm{~S} 2 \mathrm{Pd}(5 \mathrm{~nm}) / \mathrm{V}(24.2 \mathrm{~nm}) /[\mathrm{Fe}(3 \mathrm{ML}) /$ $\mathrm{V}(12 \mathrm{ML})]_{25}$, and $\mathrm{S} 3$ spin valve sample $3 \mathrm{~g}$ (see Table I). The data are offset along the intensity axis for clarity.

Superlattice reflections from the $[\mathrm{Fe} / \mathrm{V}]$ superlattice are well resolved up to at least third order. Additionally, well pronounced Kiessig fringes from the total thickness of the superlattice are visible, indicating sharp interfaces with weak interdiffusion and low roughness. ${ }^{27} \mathrm{~A}$ fit with the Parratt formalism ${ }^{28}$ gave a root mean square (RMS) interface roughness parameter of $\sigma=0.2 \mathrm{~nm}$, comparable to earlier reports on similar $[\mathrm{Fe} / \mathrm{V}]_{n}$ superlattice structures. ${ }^{25,29-34}$

In Fig. 2 we display Bragg scans of the same three samples in the angular range of the $\mathrm{MgO}$ (002) Bragg peak, where the main Bragg reflections of the epitaxial layers are expected. On the high angle side of the (002) substrate Bragg reflection there appears the (002) Bragg reflection from the thick superconducting $V$ layer at $Q_{z}=41 \mathrm{~nm}^{-1}$, in perfect agreement with the bulk lattice parameter of $V$, and the (002) superlattice Bragg reflection shifted to slightly larger $Q_{z}$ values due to the out-of-plane lattice compression in the superlattice. ${ }^{35}$ The zeroth-order superlattice peak is accompanied by many satellites on both sides, indicative of coherent growth of the superlattice.

For sample S3 there appears, in addition, the $\mathrm{Co}(11-20)$ Bragg reflection at $Q_{z}=49.40 \mathrm{~nm}^{-1}$, slightly shifted compared to bulk hcp Co (Ref. 36) with the (11-20) peak at $Q_{z}$

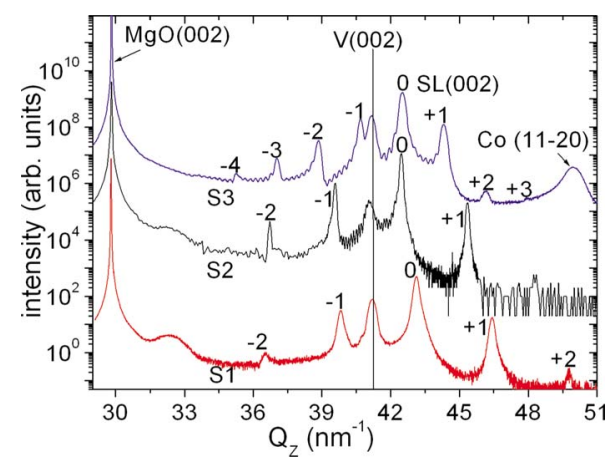

FIG. 2. (Color online) Bragg scans for the same samples of Fig. 1. $\mathrm{S} 1 \mathrm{Pd}(5 \mathrm{~nm}) / \mathrm{V}(24.2 \mathrm{~nm}) /[\mathrm{Fe}(5 \mathrm{ML}) / \mathrm{V}(8 \mathrm{ML})]_{25}, \mathrm{~S} 2$ $\mathrm{Pd}(5 \mathrm{~nm}) / \mathrm{V}(24.2 \mathrm{~nm}) /[\mathrm{Fe}(3 \mathrm{ML}) / \mathrm{V}(12 \mathrm{ML})]_{25}$, and $\mathrm{S} 3$ spin valve sample $3 \mathrm{~g}$ (see Table I). The data are offset along the intensity axis for clarity.

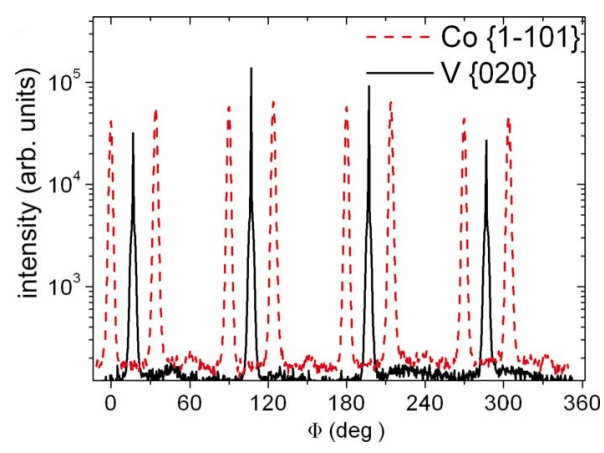

FIG. 3. (Color online) In-plane rocking scans of sample $3 \mathrm{~g}$ from Fig. 1 for the Bragg reflections $V\{200\}$ (solid line) and $\operatorname{Co}(1-101)$ (dashed line).

$=49.86 \mathrm{~nm}^{-1}$. From the half width at half maximum (FWHM) $\Delta Q$ one can calculate the out-of-plane structural coherence length $\mathrm{L}_{\text {coh }}$ of the different layers using $\mathrm{L}_{\text {coh }}$ $=\frac{2 \pi}{\Delta Q_{Z}}$ (see Ref. 37). We get a value of about $90 \%$ of the thickness for the $[\mathrm{Fe} / \mathrm{V}]$ superlattice, $80 \%$ for the thick $V$ layer, and $90 \%$ for the Co layer. The structural coherence lengths comparable to the film thicknesses indicate a high quality, coherent growth of all layers.

The in-plane single crystalline quality of the thin-film heterostructure was studied by in-plane rocking scans at grazing incidence of the $\mathrm{x}$-ray beam, ${ }^{38}$ keeping the detector angle fixed at $2 \Theta$ of the corresponding Bragg peak while rotating the sample in-plane by $360^{\circ}$ around the surface normal. A corresponding scan for one sample (sample S3 in Figs. 1 and 2 ) is depicted in Fig. 3. One observes four $V\{200\}$ Bragg reflections from the thick $V$ film separated by $\Delta \Phi=90^{\circ}$, giving clear evidence of the fourfold symmetry of the [100] axis, i.e., the epitaxial quality of the $V$ film. The in-plane Co(1-101) reflection, also shown in Fig. 3, exhibits eight well-defined peaks with a pair of peaks separated by $45^{\circ}$ accompanying each $\mathrm{V}(020)$ reflection. The origin of this pseudocubic in-plane symmetry of the Co-layer is known to result from two types of crystalline domains formed during the epitaxial growth of hcp Co on bcc V. ${ }^{36,39}$

\section{B. Superconducting parameters}

As pointed out in Sec. I, for the superconducting spin valve effect a large superconducting coherence length $\xi_{s}$ in the $V$ layer, i.e., a large electron mean-free path, is essential. We thus optimized the growth of the thick $V$ film by systematically varying the composition of the $[\mathrm{Fe} / \mathrm{V}]$ superlattice. In the series 1 samples (Table I) the thickness of the $V$ interlayer in the superlattice was fixed at $8 \mathrm{ML}$, corresponding to a thickness of $d_{V}=1.2 \mathrm{~nm}$; the thickness of the superconducting $V$ layer was fixed at $\mathrm{d}_{\mathrm{S}}=24.2 \mathrm{~nm}$. As shown by Hjörvarsson et al. ${ }^{40}$ for 8-ML-thick $V$ interlayers the interlayer exchange coupling between the Fe layers is ferromagnetic. In Fig. 4 we display the superconducting transition temperature $T_{\mathrm{S}}$ as a function of the Fe-layer thickness $d_{\mathrm{Fe}}$. One observes a curve very similar to those reported before for polycrystalline $\mathrm{Fe} / \mathrm{V} / \mathrm{Fe}$ trilayers or $\mathrm{Fe} / \mathrm{V}$ bilayers by Aarts et al. ${ }^{21}$ and Garifullin et al. ${ }^{41}$ with a shallow minimum 

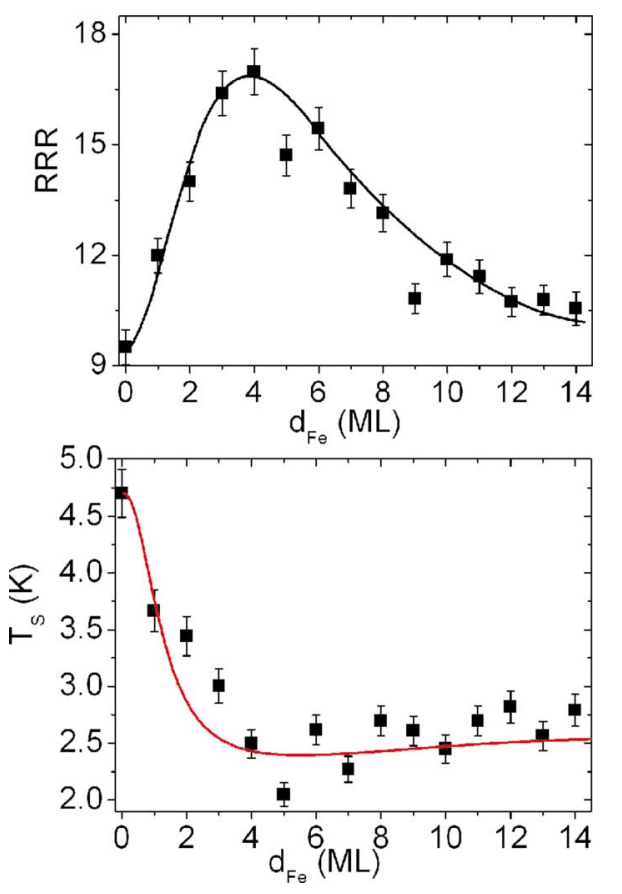

FIG. 4. (Color online) Residual resistivity ratio (upper panel) and superconducting transition temperature (lower panel) as functions of the Fe-layer thickness measured in units of monolayers for the samples $\mathrm{Pd}(5 \mathrm{~nm}) / \mathrm{V}(24.2 \mathrm{~nm}) /\left[\mathrm{Fe}\left(d_{\mathrm{Fe}}\right)(\mathrm{ML}) / \mathrm{V}(8 \mathrm{ML})\right]_{25}$. The solid line in the upper panel is a guide to the eye. The solid line in the lower panel is a theoretical fit following the theory of Tagirov (Ref. 13) with the parameters given in the main text.

in $T_{S}\left(d_{\mathrm{Fe}}\right)$ at five Fe monolayers $(0.7 \mathrm{~nm})$ and leveling off to a constant $T_{\mathrm{S}}$ for more than eight $\mathrm{Fe}$ monolayers. Interestingly, also for the superlattice with one monolayer of Fe we find a clear drop of $T_{\mathrm{S}}$ compared to the single $V$ film, although for this composition the $[\mathrm{Fe} / \mathrm{V}]$ superlattice is nonmagnetic, as shown theoretically by Mirbt et $a l^{42}$ and experimentally by Labergerie et al. ${ }^{43}$ This shows that even the nonmagnetic one-monolayer Fe-film effectively suppresses the superconducting pair density at the interface between the $V$ film and the $[\mathrm{Fe} / \mathrm{V}]$ superlattice and acts as a weak link.

The residual resistivity ratio (RRR), defined as the ratio at room temperature and $10 \mathrm{~K}$, for the samples of series 1 has also been plotted in Fig. 4. The RRR plotted refers to the thick superconducting $V$ film alone, the values measured experimentally for the whole multilayer stack are smaller, since the total resistance of the sample is combined of the superlattice resistance and the thick $V$ film connected in parallel. The RRR of the superlattice is of the order of $R R R=2$, nearly independent of the thickness combination. ${ }^{44}$ Thus, we have calculated the RRR of the thick $V$ film given in Fig. 4 by correcting the measured RRR with the conductivity contribution from the superlattice as given by Granberg et al. ${ }^{44}$ The maximum in Fig. 4 reaches a value of about $R R R=17$, this is much larger than the best RRR values obtained before in textured, polycrystalline Fe/V thin-film systems of comparable thickness, ${ }^{23}$ which typically are of the order RRR $=3-6$. The superconducting correlation length $\xi_{\mathrm{S}}$ can be calculated by the dirty limit formula

$$
\xi_{\mathrm{S}}=\frac{\pi k_{B}}{e} \sqrt{\frac{(\mathrm{RRR}-1) \xi_{\mathrm{BCS}}}{3.4 v_{F} \gamma \varrho_{\mathrm{ph}}}},
$$

with the BCS coherence length $\xi_{\mathrm{BCS}}$, the Fermi velocity $v_{F}$, the electronic specific-heat coefficient $\gamma$, and the phonon resistance $\varrho_{\mathrm{ph}}$. Using this equation with the RRR taken from Fig. 4 and the other parameters taken from the literature ${ }^{45,46}$ we get $\xi_{S}=19.4 \mathrm{~nm}$ for $\mathrm{RRR}=17$ and $\xi_{\mathrm{S}}=15.3 \mathrm{~nm}$ for $\mathrm{RRR}=11$. For the ratio $\xi_{\mathrm{S}} / d_{\mathrm{S}}$ we reach values of up to $\xi_{\mathrm{S}} / d_{\mathrm{S}}=0.80$, i.e., definitely larger than reported in the literature before, ${ }^{16-19}$ which gives an encouraging perspective for a possible improvement of the spin valve effect.

The RRR of the $V$ layer in Fig. 4 reaches a maximum at a ratio of $1: 2$ for the thickness of the Fe layer and the $V$ layer in the superlattice. Thus it seems that at this thickness ratio the growth conditions of the superconducting $V$ layer are optimized and that the defect concentration is minimized. Optimum superconducting parameters of the thick superconducting $V$ layer are thus achieved for a $[\mathrm{Fe}(4 \mathrm{ML}) / \mathrm{V}(8 \mathrm{ML})]$ or a $[\mathrm{Fe}(3 \mathrm{ML}) / \mathrm{V}(8 \mathrm{ML})]$ superlattice, a thickness combination which we will use for the investigation of the superconducting spin valve effect below.

We have fitted the $T_{\mathrm{S}}\left(d_{\mathrm{Fe}}\right)$ curve in Fig. 4 by using the theory of $\mathrm{S} / \mathrm{F}$ proximity effect as developed by Tagirov, ${ }^{13}$ which explicitly includes a finite interface transparency. Besides geometrical parameters known from the experiments and the value of $\xi_{\mathrm{S}}$ (see Table I), $T_{\mathrm{S}}\left(d_{\mathrm{Fe}}\right)$ depends on the penetration depth of the superconducting pairing function into the ferromagnet $\xi_{\mathrm{F}}$ and the interface transparency parameter $T$. For both parameters we derived $\xi_{\mathrm{F}}=0.62 \mathrm{~nm}$ and $T=0.68$ from the fit, this is similar to the values obtained before for $\mathrm{Fe} / \mathrm{V}$ interfaces. ${ }^{2,47}$

\section{S/F1/N/F2-type superconducting spin valves}

We prepared a second series of samples with the Fe thickness kept constant at $3 \mathrm{ML}$ and varying the $V$ thickness in the superlattice between $8 \mathrm{ML}$ and $15 \mathrm{ML}$ (series 2 in Table I) thus covering the thickness range between $n=11$ and $n=13$ where Hjörvarsson et al. ${ }^{40}$ observed antiferromagnetic interlayer exchange coupling. The superconducting transition temperature of all these samples was about $T_{\mathrm{S}}=3 \mathrm{~K}$ with a scatter of about $0.2 \mathrm{~K}$. In the most interesting range with antiferromagnetic coupling the samples have a $\mathrm{RRR} \approx 11$, definitely larger than in our previous series of samples. ${ }^{23}$ Only the sample with $n=12$ exhibits perfect antiferromagnetic order with vanishing remanent magnetization, as seen in the magnetization curve Fig. 5(a) measured at $10 \mathrm{~K}$ and consistent with previous results reported in the literature. ${ }^{48}$ The ferromagnetic saturation field of the Fe sublattices is about $2.5 \mathrm{kOe}$.

In this sample the magnetization direction of successive Fe layers in the superlattice can be gradually rotated from an antiparallel alignment in zero field to a parallel alignment for fields above $2.5 \mathrm{kOe}$. The upper critical magnetic field for the direction parallel to the film plane is plotted in Fig. 5(b). The transition temperature $T_{\mathrm{S}}$ has been defined as the $50 \%$ value of the resistance. Other possible definitions, as the $10 \%$ value or the extrapolated down set of the resistance give identical curves, only slightly shifted along the $T$ axis. 

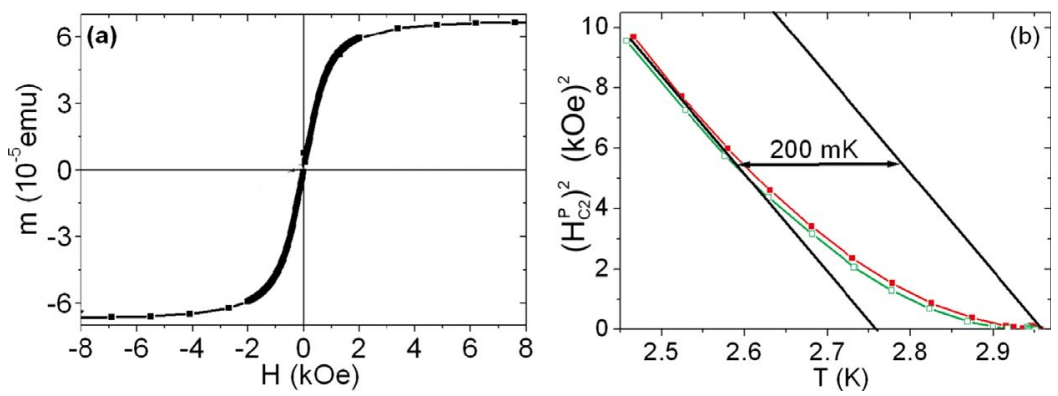

FIG. 5. (Color online) (a) Magnetic moment versus magnetic field measured at $10 \mathrm{~K}$ and (b) squared parallel upper critical magnetic field versus temperature. Red line (filled squares): field up; green line (unfilled squares): field down for the sample $\mathrm{Pd}(5 \mathrm{~nm}) / \mathrm{V}(24 \mathrm{~nm}) /[\mathrm{Fe}(3 \mathrm{ML}) / \mathrm{V}(12 \mathrm{ML})]_{25}$ of series 2 (see Table I). Solid lines indicate the upper critical fields expected for perfect parallel alignment (left line) and antiparallel alignment (right line) of the Fe layers in the superlattice. The difference in the transition temperature is $200 \mathrm{mK}$.

For a two-dimensional thin superconducting film the classical result for the temperature dependence of the parallel upper critical field close to $T_{\mathrm{S}}$ is (see, e.g., Klemm et $a l^{49}$ )

$$
H_{C 2}^{\mathrm{P}}(T)=\frac{\Phi_{0}}{2 \pi \xi_{0}} \frac{\sqrt{12}}{d_{\mathrm{S}}} \sqrt{\left(1-\frac{T}{T_{\mathrm{S}}}\right)},
$$

where $\Phi_{0}$ is the flux quantum, $d_{\mathrm{S}}$ is the thickness of the $\mathrm{S}$ film, and $\xi_{0}$ the Ginzburg-Landau correlation length, which is related to Pippards correlation length $\xi_{\mathrm{S}}$ listed in Table I by $\xi_{0}=1.6 \xi_{\mathrm{s}}$.

In the temperature dependence of the upper critical field depicted in Fig. 5(b) we notice an increasing deviation from the straight-line behavior at low fields, starting at the ferromagnetic saturation field of the superlattice at about $2.5 \mathrm{kOe}$. This deviation must be attributed to the rotation of the magnetization direction of successive Fe layers in the superlattice from parallel to antiparallel. Extrapolating the transition temperatures as shown in Fig. 5(b) one finds a difference $\Delta T_{\mathrm{S}}$ of $200 \mathrm{mK}$ for successive Fe layers being in the antiparallel and the parallel orientations, respectively. This is the superconducting spin valve effect calculated for the S/F1/N/F2 structure by $\mathrm{Oh}$ et al. in Ref. 24 and reported for the $[\mathrm{Fe}(2 \mathrm{ML}) / \mathrm{V}(11 \mathrm{ML})]_{25} / \mathrm{V}$ systems in Ref. 23 . The maximum shift $\Delta T_{\mathrm{S}}$ we have observed in Ref. 23 was about 120 $\mathrm{mK}$, definitely smaller than for the present sample. We think that this reflects the improved structural and electronic qualities of our samples with $\xi_{\mathrm{S}} / d_{\mathrm{S}}=0.67$ for the sample in Fig. 5(b) compared to a maximum value of $\xi_{\mathrm{S}} / d_{\mathrm{S}}=0.4$ for the previous samples. $^{23}$

\section{F1/S/F2-type superconducting spin valves}

We now turn to the main issue of the present paper, namely, the superconducting spin valves of type F1/S/F2. In Fig. 6(a) we show the magnetic hysteresis loop measured at $5 \mathrm{~K}$ of sample $3 \mathrm{a}$ from Table $\mathrm{I}$. This is a spin valve with a 7 $\mathrm{nm}$ thick $\mathrm{Co} / \mathrm{CoO}$ ferromagnetic top layer. The hysteresis loop has been measured after field cooling in an applied field of $6 \mathrm{kOe}$ in order to establish an exchange bias shift of the coercive field. The coercive fields of the $[\mathrm{Fe} / \mathrm{V}]$ superlattice and of the Co layer are distinctly different, namely, about 0.1 and $2.2 \mathrm{kOe}$, respectively, giving a broad range of fields with an essentially antiparallel orientation of the magnetization direction of F1 and F2, which is the plateau region on the positive field side in the hysteresis loop in Fig. 6(a). However, one should notice that in the plateau region the F1 and F2 layers are not in a perfect single domain state, since the $[\mathrm{Fe} / \mathrm{V}]$ superlattice is only magnetically saturated above the saturation field of about $0.5 \mathrm{kOe}$ and the magnetization of the Co layer already deviates from ferromagnetic saturation below about $3 \mathrm{kOe}$. Before coming to the temperature dependence of the upper critical field plotted in Fig. 6(b), we first must discuss the field dependence of the resistive transition curves, since a precise determination of the superconducting transition temperature from the resistivity curves $R(T)$ is not trivial.

Characteristic examples for resistive transitions on different branches of the hysteresis loop are shown in Fig. 7. In Figs. 7(a)-7(c) we have plotted the transitions at positive and negative magnetic fields close to the coercive field of the $[\mathrm{Fe} / \mathrm{V}]$ superlattice at $H_{c} \approx 0.1 \mathrm{kOe}$. One sees that at low fields $[H= \pm 11$ Oe, Fig. $7(\mathrm{a})]$ both transition curves are vir-
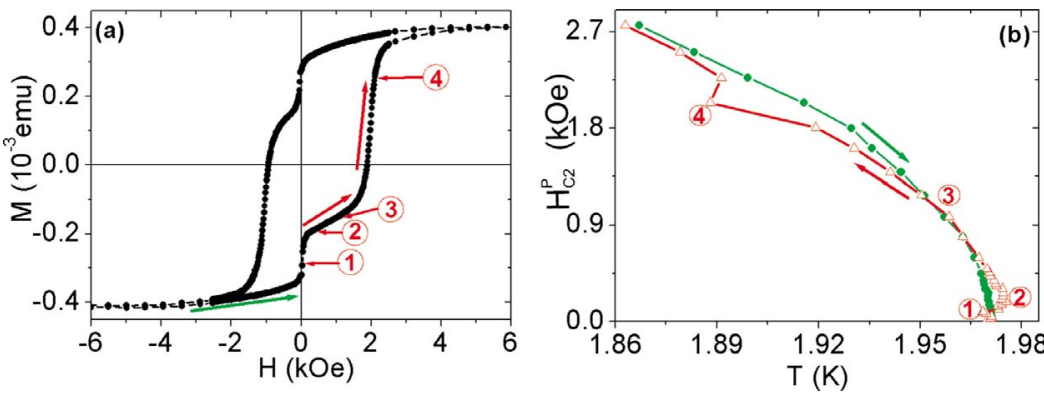

FIG. 6. (Color online) (a) Hysteresis loop measured at $5 \mathrm{~K}$ and (b) upper critical magnetic field for sample $3 \mathrm{a}$ (see Table I). The direction of the arrows defines the sequence of field change during the measurements of $H_{C 2}^{\mathrm{P}}(T)$ along the descending branch of the hysteresis loop. Filled dots (green curve): negative fields; empty triangles (red curve): positive fields. Numbers 1-4 in the figure relate to comments in the main text (colored online). 

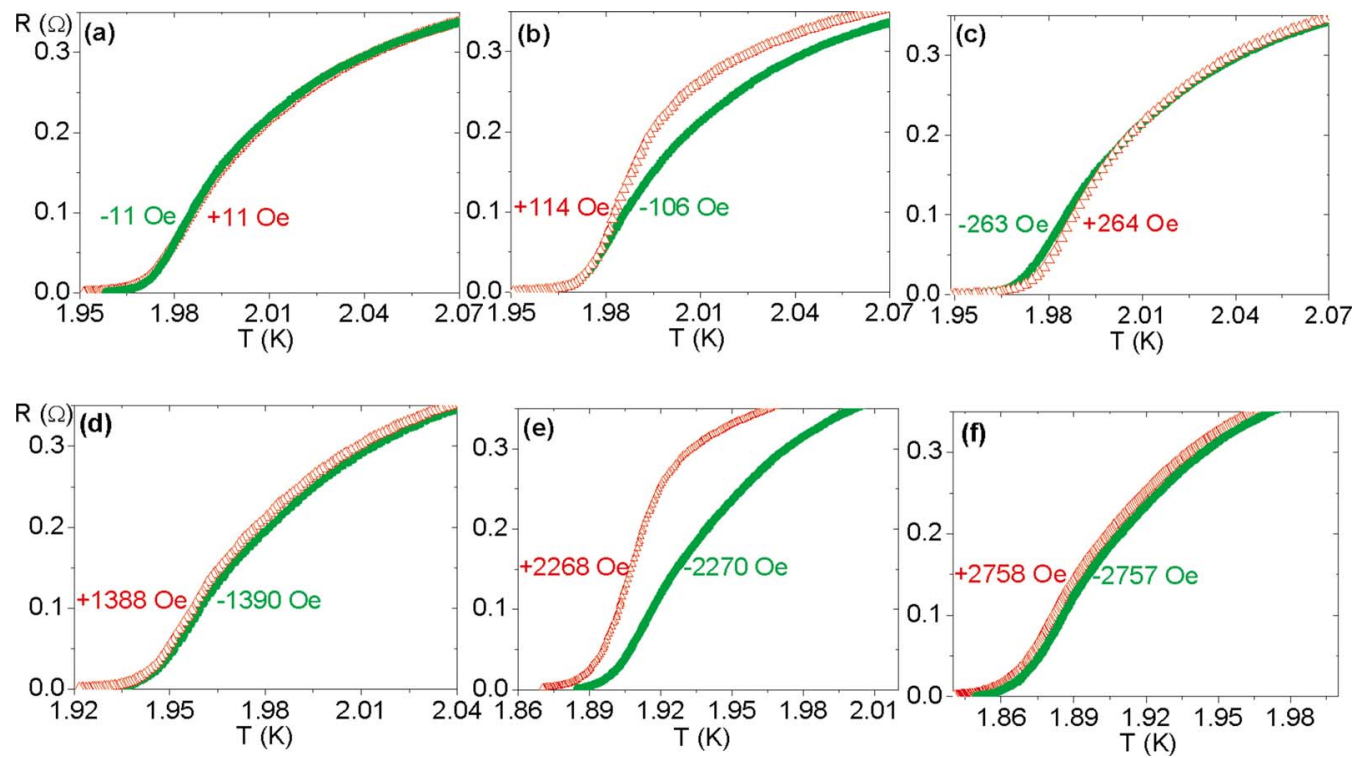

FIG. 7. (Color online) (a)-(c) Resistive transition for sample 3a from Fig. 6 at different fields close to the coercive field of the [Fe/V] superlattice and (d)-(f) close to the coercive field of the Co layer for positive and negative fields. For the sequence of fields see the arrows in Fig. 6(b). Filled dots (green curve): negative fields; empty triangles (red curve): positive fields.

tually identical. Just above the coercive field $H_{c}[H$ $= \pm 110$ Oe, Fig. 7(b)], however, the shape of the transition curves is different. For $H=+110$ Oe with the $[\mathrm{Fe} / \mathrm{V}]$ superlattice just remagnetizing, the transition width is definitely narrower. At higher fields $[H= \pm 264$ Oe, Fig. $7(\mathrm{c})]$ with the $[\mathrm{Fe} / \mathrm{V}]$ superlattice close to magnetic saturation in the direction antiparallel to the Co layer the two resistive transition curves become very similar again, although with a small shift of the down-set temperature toward higher temperatures for the positive field. It is plausible to associate the changed shape of the transition curve in Fig. 7(b) to the magnetic stray fields from the domains in the multidomain state of the $[\mathrm{Fe} / \mathrm{V}]$ superlattice, as discussed previously in Ref. 20. The stray fields mainly suppress superconducting fluctuations and superconducting weak links, thus causing a narrowing of the resistive transition.

Since the shape of the transition curve changes, an unambiguous definition of the superconducting transition temperature is critical. The best definition of $T_{s}$ is the extrapolated down-set temperature of the resistivity, since it indicates the onset of bulk superconductivity. With this definition one sees that in Fig. 7(b) the bulk $T_{s}$ is not affected by the stray fields, the down-set temperature is virtually identical for both field directions.

In Figs. 7(d)-7(f) we have also plotted the resistivity curves close to the coercive field of the Co film at $H_{c}$ $\approx 2.1 \mathrm{kOe}$. One recognizes a similar sequence of curves as in Figs. 7(a)-7(c) with the shape of the transition curve being nearly identical far above and far below $H_{c}$ [Figs. 7(d) and 7(f)], but strongly changed just above $H_{c}$ [Fig. 7(e)] in the multidomain state of the Co film. Comparing the resistive transitions in Figs. 7(b) and 7(e) in the multidomain state of either $\mathrm{Co}$ or the $[\mathrm{Fe} / \mathrm{V}]$ superlattice one notices that obviously the stray fields from the Co film affect the superconducting transition much more that those from the $[\mathrm{Fe} / \mathrm{V}] \mathrm{su}-$ perlattice. In part this may be explained by the different thicknesses ( $7 \mathrm{~nm}$ Co compared to about $4.5 \mathrm{~nm}$ Fe in the superlattice) and the different magnetic moments $\left(1.7 \mu_{B}\right.$ per $\mathrm{Co}$ atom and $1.2 \mu_{B}$ per $\mathrm{Fe}$ atom $\left.{ }^{50}\right)$. But we believe that the main reason is a different magnetic domain structure and a correspondingly different geometry of the stray fields emanating from the $[\mathrm{Fe} / \mathrm{V}]$ or the $\mathrm{Co}$ layer. In Co-layers exchange biased by $\mathrm{CoO}$ the magnetic domains can be very small $^{26}$ and the strength of stray field increases with decreasing domain size. In Ref. 51 a typical ripple domain structure with very fine stripe domains is reported for the $\mathrm{Co} / \mathrm{CoO}$ system. Contrary to the situation at the magnetic reversal of the $[\mathrm{Fe} / \mathrm{V}]$ superlattice, the stray fields from the Co layer even shift the down set of the resistivity, i.e., the bulk $T_{s}$, to lower temperatures. This maximum shift is observed close to the coercive field of Co.

The upper critical field for the field direction parallel to the film plane, determined from the extrapolated down set of the resistivity as just discussed, is plotted in Fig. 6(b) for positive and negative fields. For a single superconducting film both curves should coincide. The deviations between the two curves actually observed in Fig. 6(b) originate from the stray fields of the ferromagnetic layers F1 and F2, as just discussed, and the superconducting spin valve effect. The measurements in Fig. 6(b) start at high negative magnetic fields and then proceed along the ascending magnetization branch toward high positive fields. At positive fields the F1 and F2 layers are remagnetized [see hysteresis loop in Fig. 6(a)], first from a parallel to an antiparallel orientation of F1 and $\mathrm{F} 2$ at $H \approx 0.1 \mathrm{kOe}$ and then to a parallel orientation at $H \approx 2.1 \mathrm{kOe}$. For negative fields $H_{C 2}^{\mathrm{P}}(T)$ decreases monotonously with increasing temperature and, to a good approximation, follows the standard two-dimensional $H_{C 2}^{\mathrm{P}}(T)$ $\propto \sqrt{\left(T-T_{\mathrm{S}}\right)}$ power law [Eq. (2)]. For positive fields during the magnetization reversal of the F1 and F2 layers, however, one observes a strongly anomalous curve with an unusual nonmonotonous $T$ dependence close to the coercive fields of F1 

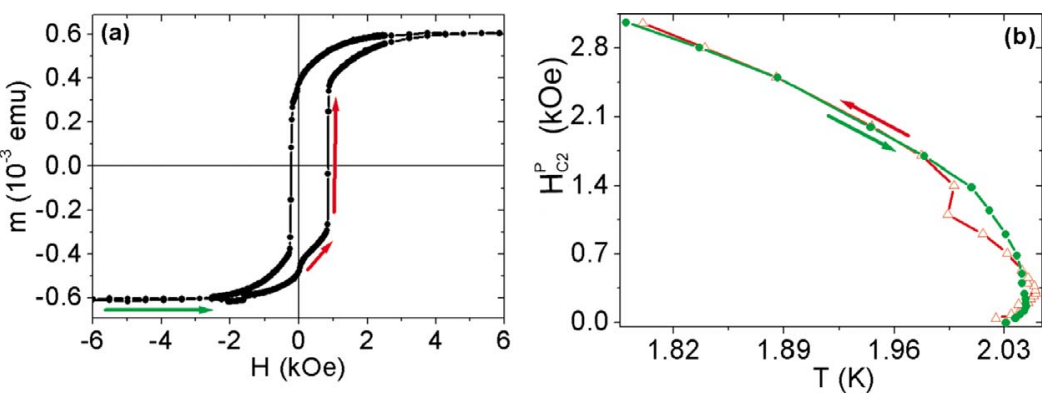

FIG. 8. (Color online) (a) Hysteresis loop measured at $5 \mathrm{~K}$ and (b) parallel upper critical magnetic field for sample $3 \mathrm{~b}$ (see Table I). The two branches of $H_{C 2}^{\mathrm{P}}(T)$ are defined as in Fig. 6 . Filled dots (green curve): negative fields; empty triangles (red curve): positive fields. and F2. This is caused by a competing influence of the superconducting spin valve effect and the magnetic stray fields from $\mathrm{F} 1$ and F2. With the magnetization reversal of the $\mathrm{Fe} / \mathrm{V}$ superlattice [between points 1 and 2 in Fig. 6(b)] the antiparallel orientation of F1 and F2 is gradually established and the superconducting transition temperature increases due to the superconducting spin valve effect. The maximum positive shift of $T_{\mathrm{S}}$ measured at $0.3 \mathrm{kOe}$ is $\Delta T_{\mathrm{S}} \approx 5 \mathrm{mK}$. Toward higher fields the two branches of the $H_{C 2}^{\mathrm{P}}(T)$ approach each other again and cross at about 1 kOe [point 3 in Fig. 6(b)]. This is caused by the domain formation and the stray fields from the Co film. This negative shift $\Delta T_{\mathrm{S}}$ reaches a maximum of $\Delta T_{\mathrm{S}}=-30 \mathrm{mK}$ at the coercive field of the Co layer [point 4 in Fig. 6(b)]; above this field the magnetic domains in the Co film vanish gradually and the two branches in Fig. 6(b) approach each other again.

In Figs. 8(a) and 8(b) we show the hysteresis loop and the parallel upper critical field of a second spin valve sample, this time with the top layer F2 replaced by a $5 \mathrm{~nm}$ thick Fe-film exchange coupled to a further $\mathrm{Co} / \mathrm{CoO}$ bilayer (sample $3 \mathrm{~b}$ from Table I). The superconducting $V$ layer has a thickness of $35 \mathrm{~nm}$ as compared to $24 \mathrm{~nm}$ for the sample shown in Fig. 6. Nevertheless, both samples have a nearly identical $T_{S} \approx 2 \mathrm{~K}$, since the increase in thickness is compensated by a stronger pair breaking field of the Fe layer. The hysteresis curve in Fig. 8(a) reveals that the coercive field of the $\mathrm{Fe} / \mathrm{Co}$ top layer is shifted to $H_{c} \approx 1 \mathrm{kOe}$; thus an approximately antiparallel orientation of $\mathrm{F} 1$ and $\mathrm{F} 2$ is expected between $H \approx 0.2$ and $1 \mathrm{kOe}$.

The two branches of the upper critical field $H_{C 2}^{\mathrm{P}}(T)$ in Fig. 8(b) are qualitatively very similar to those discussed for the sample of Fig. 6(b) and can be explained following the same lines. The strong anomaly for positive fields at $H \approx 1 \mathrm{kOe}$ correlates with the coercive field of the Fe/Co layer. Actually, for a narrow field range between 0.3 and $0.5 \mathrm{kOe}$ the superconducting transition temperature for positive fields is slightly larger than for negative fields, the maximum difference is $+4 \mathrm{mK}$. We would attribute this small shift to the superconducting spin valve effect. At variance to the behavior in Fig. 6(b), we here observe an anomalous $H_{C 2}^{P}(T)$ curve with a positive slope at low fields for positive as well as for negative fields. This is caused by magnetic domain formation of the $[\mathrm{Fe} / \mathrm{V}]$ superlattice at low fields.

The two spin valves just discussed indicate that using Co and Fe layers as the F2 layer the spin valve effect exists, but is very small, only of the order of $+5 \mathrm{mK}$. This small positive shift of $T_{s}$ is difficult to resolve unambiguously above the background of the negative shift of $T_{\mathrm{S}}$ induced by the magnetic stray fields. The main reason for this small shift is most likely the rather small ratio $\xi_{\mathrm{S}} / d_{\mathrm{S}}$ (see Table I) and the relatively small interface transparency. We have tried to improve the $\xi_{\mathrm{S}} / d_{\mathrm{S}}$ ratio by decreasing the $V$ thickness further, but this possibility is limited because with the strong pair breaking in the $\mathrm{Fe}$ and $\mathrm{Co}$ films the critical thickness for superconductivity is already nearly reached for the samples just discussed.

We thus followed a different route for enhancing the spin valve effect and weakened the exchange interactions by replacing the concentrated magnetic elements $\mathrm{Co}$ and $\mathrm{Fe}$ in the $\mathrm{F} 1$ and F2 layers by $\mathrm{Fe}_{x} \mathrm{~V}_{1-x}$ alloy layers (see Table I). Epitaxial superlattices $\left[\mathrm{V} / \mathrm{Fe}_{x} \mathrm{~V}_{1-x}\right]$ and $\left[\mathrm{Fe} / \mathrm{Fe}_{x} \mathrm{~V}_{1-x}\right]$ can be prepared on $\mathrm{MgO}(100)$ with a quality similar to that of $[\mathrm{Fe} / \mathrm{V}]$, as shown recently by Skubic et al. ${ }^{52}$ The alloy system $\mathrm{Fe}_{x} \mathrm{~V}_{1-x}$ forms a homogeneous solid solution down to the critical concentration of spontaneous ferromagnetism close to $x=0.22 .^{21,52}$ The atomic magnetic moment of $\mathrm{Fe}$ decreases approximately linearly with the Fe concentration ${ }^{21}$ and the magnetic exchange interaction is expected to decrease correspondingly. The weakening of the exchange interaction should decrease the critical thickness for superconductivity in the $V$ layer and thus enable larger $\xi_{\mathrm{S}} / d_{\mathrm{S}}$ values and simultaneously enhance the interface transparency. Actually in the spin valves with $\mathrm{Fe}_{x} \mathrm{~V}_{1-x}$ alloy layers we have reached $\xi_{\mathrm{S}} / d_{\mathrm{S}}$ ratios of up to $\xi_{\mathrm{S}} / d_{\mathrm{S}} \approx 0.67$, definitely larger than when using concentrated Fe or Co layers (see Table I).

In Fig. 9(a) we show the hysteresis loop and the upper critical field of a sample with a $\mathrm{Fe}_{24} \mathrm{~V}_{76}$ alloy layer in the superlattice and as the top ferromagnetic layer (sample $3 \mathrm{~h}$ from Table I). The hysteresis loop again shows a two-step magnetization reversal process. The alloy superlattice magnetization reversal occurs at fields below $0.3 \mathrm{kOe}$ and the $\mathrm{Fe}_{24} \mathrm{~V}_{76}$ top layer, exchange coupled to the $\mathrm{Co} / \mathrm{CoO}$-bilayer, remagnetizes above $1.5 \mathrm{kOe}$. In Figs. 10(a)-10(f) we have plotted a series of superconducting resistive transitions for this sample close to the coercive fields of both magnetic layers. Remarkably, for this series of measurements one notices no change in the shape of the superconducting transition caused by the stray fields in the multidomain state of the F1 and F2 layers [Figs. 10(b) and 10(e)], much unlike the situation in Fig. 7. The transition is merely shifted along the $T$ axis, keeping its shape and width constant. One can clearly see the spin valve effect as a shift of the transition by $\Delta T_{\mathrm{S}}$ $\approx 12 \mathrm{mK}$ in the whole field range of the plateau between 0.3 and $1.5 \mathrm{kOe}$ in Fig. 9(a).

The vanishing influence of the magnetic dipolar field from the F1 and F2 layers $\mathrm{Fe}_{1-x} \mathrm{~V}_{x}$ is on one hand due to the smaller volume magnetization $M$ of the alloy layers. The small atomic magnetic moment per Fe atom [about $0.1 \mu_{B}$ in 

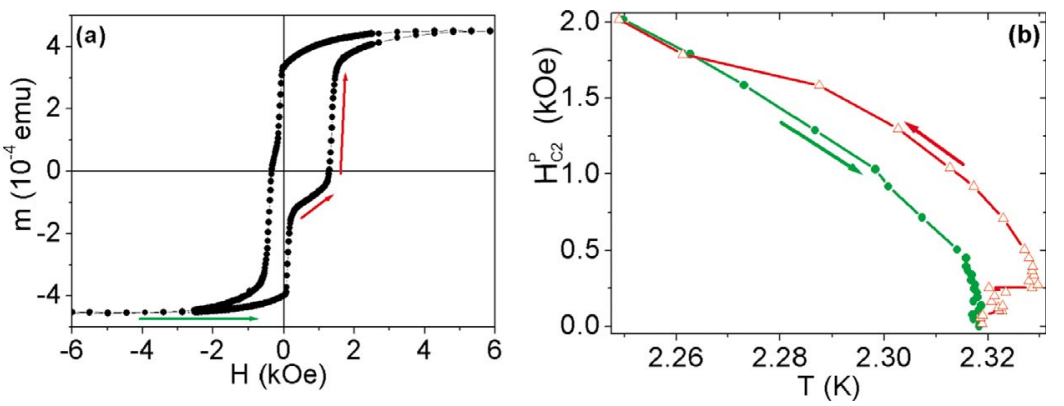

FIG. 9. (Color online) (a) Hysteresis loop measured at $5 \mathrm{~K}$ and (b) parallel upper critical magnetic field for sample $3 \mathrm{~h}$ (see Table I). The two branches of $H_{C 2}^{\mathrm{P}}(T)$ are defined as in Fig. 6. Filled dots (green curve): negative fields; empty triangles (red curve): positive fields.

$\mathrm{Fe}_{24} \mathrm{~V}_{76}$ (Ref. 21)] and the magnetic dilution give rise to a reduction in $M$ by more than a factor of 10 compared to pure Fe and a corresponding decrease in the strength of the dipolar stray fields. A modified magnetic domain structure might also play a role. Interestingly, the stray field from the $7 \mathrm{~nm}$ Co film on top of the $\mathrm{Fe}_{24} \mathrm{~V}_{76}$ alloy layer also does not affect the shape of the resistive transition [Fig. 10(e)], contrary to the situation with the Co layer deposited directly on the superconducting $V$ layer. Thus the soft ferromagnetic $\mathrm{Fe}_{24} \mathrm{~V}_{76}$ alloy layer between the Co layer and the $V$ layer seems to effectively screen the stray field from the Co layer.

In Figs. 11(a) and 11(b) we show another example for a spin valve with a $\mathrm{Fe}_{32} \mathrm{~V}_{68}$ alloy layer used as $\mathrm{F} 1$ and $\mathrm{F} 2$ (sample $3 \mathrm{~g}$ from Table I). For this sample we again observe a clear shift of the upper critical field curve between $H$ $\approx 0.2$ and $1.5 \mathrm{kOe}$ in the plateau region of the hysteresis loop, i.e., in the range of an antiparallel orientation of F1 and F2. For this sample the amplitude of the shift is $\Delta T_{\mathrm{S}}$ $\approx 20 \mathrm{mK}$, the largest spin valve effect we have detected during the present investigation. We consider the results presented in Figs. 9(b) and 11(b) as clear experimental evidence for the superconducting spin valve effect.

Finally, we note another positive aspect when using weakly ferromagnetic $\mathrm{Fe}_{x} \mathrm{~V}_{1-x}$ alloy layers in the superconducting spin valves. The superconducting resistive transition for these samples becomes very sharp. For the sample in Fig.
10 the transition width is only $30 \mathrm{mK}$, about a factor of 3 smaller than for the transition shown in Fig. 7. The likely reason for this is that lateral inhomogeneities in the ferromagnetic layers are less important for the superconductivity in the case of weakly ferromagnetic layers.

With the narrow superconducting transition width and the relatively large shift $\Delta T_{\mathrm{S}}$ given, the realization of an ideal superconducting switch comes within reach. According to Fig. 10 the resistivity change $\Delta R$ between parallel and antiparallel magnetizations of F1 and F2 is about $30 \%$. For an ideal superconducting switch $\Delta R=100 \%$ is desirable. The present experiments are pointing in the right direction and constitute a considerable improvement compared to earlier reports. ${ }^{15,16,18}$

In the last column of Table I we summarized the superconducting spin valve shift $\Delta T_{\mathrm{S}}$ of all samples analyzed during the present study. When comparing the different values one should consider that there are three obvious important parameters determining the magnitude of the shift. The first (trivial) one is the superconducting transition temperature $T_{s}$, which is mainly determined by the thickness of the superconducting layer. $\Delta T_{\mathrm{S}}$ increases with decreasing $T_{s}$ and should diverge for $T_{s} \rightarrow 0$, i.e., when approaching the critical thickness for superconductivity (see, e.g., Ref. 17). The second key parameter is the ratio $\xi_{\mathrm{S}} / d_{\mathrm{S}}$, as discussed in Sec. I. The third important parameter is the quantum-mechanical trans-
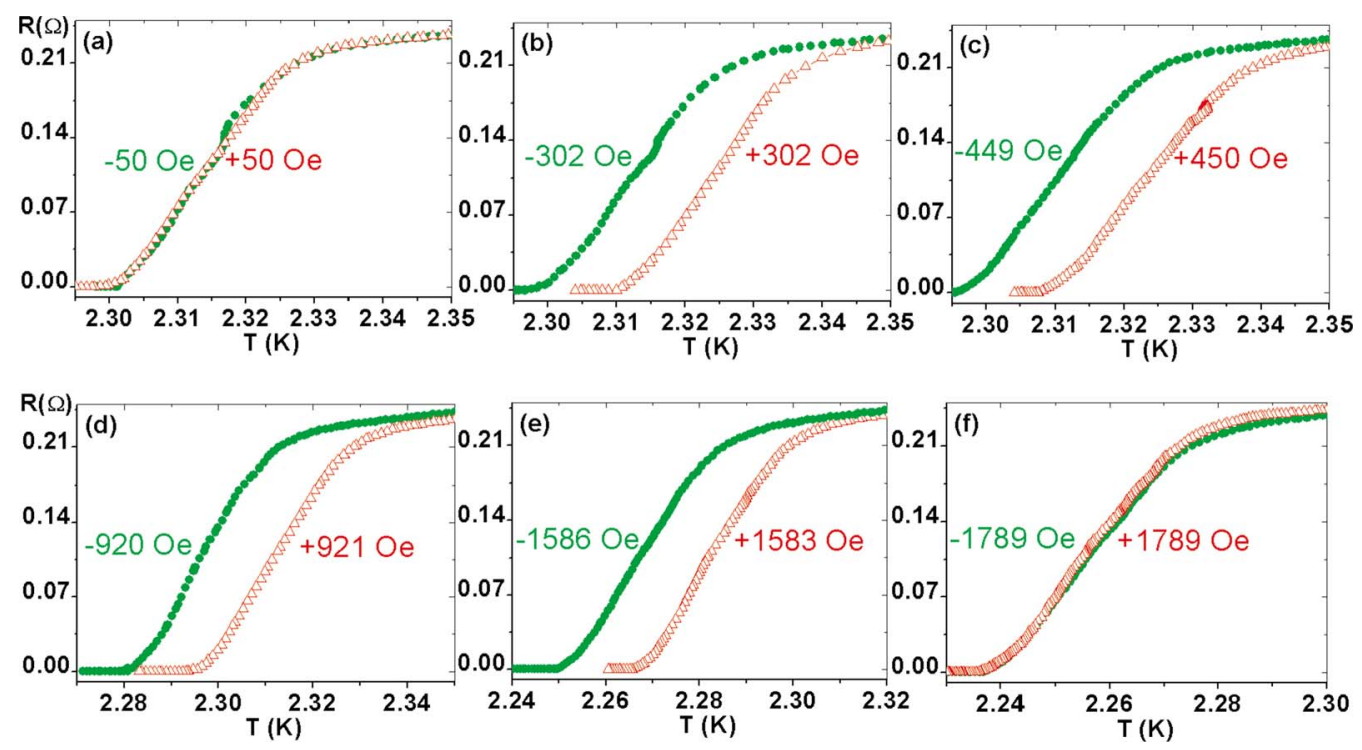

FIG. 10. (Color online) Resistive transition for the sample of Fig. 9 at different magnetic fields on the two branches in Fig. 9(b). Filled dots (green curve): negative fields; empty triangles (red curve): positive fields. 

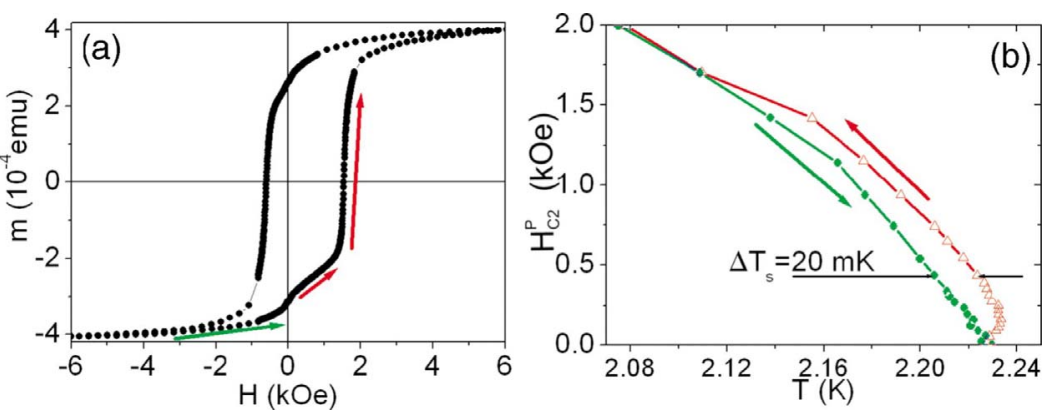

FIG. 11. (Color online) (a) Hysteresis loop measured at $5 \mathrm{~K}$ and (b) parallel upper critical magnetic field for sample $3 \mathrm{~g}$ (see Table I). The two branches of $H_{C 2}^{\mathrm{P}}(T)$ are defined as in Fig. 6. Filled dots (green curve): negative fields; empty triangles (red curve): positive fields. parency, which increases strongly when approaching the critical concentration of ferromagnetic ordering $x_{c}=0.22$ in the alloy system $\mathrm{Fe}_{x} \mathrm{~V}_{1-x}$ (see Ref. 21). Inspecting the data in Table I in detail one can qualitatively resolve the influence of the different parameters. For the spin valves with concentrated Fe and Co layers (samples 3a, 3b, and 3c) the value of $T_{s}$ dominates, the lowest $T_{s}$ (sample $3 \mathrm{c}$ with $T_{s}=1.42 \mathrm{~K}$ ) correlates with the largest $\Delta T_{\mathrm{S}}=6 \mathrm{mK}$. However, $\Delta T_{\mathrm{S}}$ is small since the transparency is small.

Decreasing the Fe concentration in $\mathrm{Fe}_{x} \mathrm{~V}_{1-x}$ the maximum shift $\Delta T_{\mathrm{S}}$ which can be obtained increases, but one needs concentrations rather close to the critical concentration for a sizable improvement. An Fe concentration $x=0.47$ (sample $3 \mathrm{~d}$ ) is not much better than pure $\mathrm{Fe}$ or Co layers, probably because the ferromagnet is still too strong and the band splitting too large. The concentration $x=0.32$ works much better and within the series of samples $3 \mathrm{e}, 3 \mathrm{f}$, and $3 \mathrm{~g}$ with $x$ $=0.32$ and different $V$ thicknesses one again recognizes $\Delta T_{\mathrm{S}}$ increasing with decreasing $T_{\mathrm{S}}$ reaching a maximum of 20 $\mathrm{mK}$ for the lowest $\left(T_{\mathrm{S}}\right)$.

A special situation is encountered when approaching the critical concentration even closer. Sample $3 \mathrm{~h}$ with $x=0.24$ has the best $\xi_{\mathrm{S}} / d_{\mathrm{S}}$ ratio of all spin valves in Table I and a comparable transition temperature, but nevertheless $\Delta T_{\mathrm{S}}$ $=12 \mathrm{mK}$ is smaller than the maximum value we obtained for $x=0.32$. We think the reason for this is that for concentrations too close to the critical concentration the formation of nonferromagnetic interlayers is highly probable, since even weak interdiffusion drives the layers into the nonferromagnetic state. Nonferromagnetic interlayers are very detrimental for the spin valve effect because they induce strong pair breaking scattering. ${ }^{13}$

\section{SUMMARY AND CONCLUSIONS}

The main aim of the present work was to investigate the superconducting spin valve effect of F1/S/F2-type spin valves with an optimum structural quality which can be realized in the epitaxial Fe/V layer systems. Actually, using weakly ferromagnetic $\mathrm{Fe}_{x} \mathrm{~V}_{1-x}$ alloy layers as $\mathrm{F} 1$ and $\mathrm{F} 2$, we observed a well-defined superconducting spin valve effect with a shift of the superconducting transition temperature of $\Delta T_{\mathrm{S}}=20 \mathrm{mK}$ and a superconducting transition temperature $T_{\mathrm{S}}=2.2 \mathrm{~K}$. The observed shift is about a factor of 2 larger than reported before in the literature for comparable transition temperatures. ${ }^{16-19}$ Thus, to this end, the high quality interfaces of the Fe/ $\mathrm{V}$ epitaxial layer system definitely enhanced the spin valve effect. We concluded from our results that in addition to the weaker ferromagnetic exchange field in the alloy layers, which helps to stabilize superconductivity down to lower thicknesses of the superconducting film, the increased interface transparency is essential.

Using pure Fe and Co layers as F1 and F2, the shift $\Delta T_{\mathrm{S}}$ we observed was much smaller, only up to $5 \mathrm{mK}$, but we could demonstrate that it does exist. We thus recovered the problem with the spin valve effect in valves with strongly ferromagnetic layers in the literature: ${ }^{16-20}$ even with the higher quality of the interfaces the spin valve effect is small. In addition, for concentrated $\mathrm{Fe}$ and Co layers used as $\mathrm{F} 1$ and F2 there is strong, competing influence from the dipolar fields of magnetic domains on the superconducting transition. This makes the small spin valve effect difficult to resolve unambiguously and might be the reason for negative results in the literature when trying to detect the spin valve effect in magnetic remanences of F1 and F2. A perfect antiparallel orientation of F1 and F2 with a single magnetic domain state for both films is difficult to achieve and residual stray fields from magnetic domains may completely mask the spin valve effect. For our samples only a careful analysis of the field dependence of the superconducting transition for both field directions revealed the existence of a small spin valve effect.

One important practical advantage when using diluted $\mathrm{Fe}_{x} \mathrm{~V}_{1-x}$ ferromagnetic alloys in the spin valves is a reduced influence of the magnetic stray fields on the superconducting transition. For the spin valves with the lowest Fe concentrations this influence virtually vanishes and the spin valve effect in its pure form, as predicted by the theory, becomes visible experimentally. This in part is simply due to the smaller magnetic induction and a corresponding reduction in the strength of the stray field, a modified magnetic domain structure with larger domains for the alloy layers is probably also important.

A final comment concerning the superconducting spin valves of S/F1/N/F2 type, which we also have discussed in this paper, seems to be in order. In our $[\mathrm{Fe} / \mathrm{V}]$-superlattice systems we use an antiferromagnetic interlayer exchange coupling to establish an antiferromagnetic orientation between two successive Fe layers and thus we cannot switch the relative magnetization direction, but only rotate it gradually from antiparallel to parallel. We observed an anomalous shift $\Delta T_{\mathrm{S}} \approx 200 \mathrm{mK}$ accompanying this rotation. For the spin valves of $\mathrm{F} 1 / \mathrm{S} / \mathrm{F} 2$ type with comparable $\xi_{\mathrm{S}} / d_{\mathrm{S}}$ ratios the shift we observed is much smaller. The model calculations in the literature ${ }^{13,15,24}$ offer no straightforward explanation for this discrepancy, one rather would expect a similar magni- 
tude of the shift for both types of valves. This might indicate the importance of the thin $V$ layer between the Fe layers in the superlattice. If the superconducting pair density of this thin $V$ layer varies strongly with the relative orientation of the magnetizations of $\mathrm{F} 1$ and $\mathrm{F} 2$, then, via the proximity effect, the superconducting transition temperature of the thick $V$ layer will also vary. This would open an alternative route for the realization of a superconducting spin valve with better performance.

\section{ACKNOWLEDGMENTS}

The authors (G.N., K.W., and H.Z.) thank the DFG for financial support within Contract No. SFB 491. M.M., A.L., and B.H. would like to acknowledge financial support from STINT, SSF, VR, and KAW. I.A.G. wants to thank the RFBR for financial support (Grant No. 08-02-00098).
*Gregor.Nowak@rub.de

${ }^{1}$ C. L. Chien and D. Reich, J. Magn. Magn. Mater. 200, 83 (1999).

${ }^{2}$ I. A. Garifullin, J. Magn. Magn. Mater. 240, 571 (2002).

${ }^{3}$ A. A. Golubov, M. Yu. Kupriyanov, and E. Il'ichev, Rev. Mod. Phys. 76, 411 (2004).

${ }^{4}$ A. I. Buzdin, Rev. Mod. Phys. 77, 935 (2005).

${ }^{5}$ F. S. Bergeret, A. F. Volkov, and K. B. Efetov, Rev. Mod. Phys. 77, 1321 (2005).

${ }^{6}$ K. B. Efetov, I. A. Garifullin, A. F. Volkov, and K. Westerholt, in Magnetic Heterostructures: Advances and Perspectives in Spinstructures and Spin-transport, edited by H. Zabel and S. D. Bader (Springer, New York, 2008), Vol. 227.

${ }^{7}$ M. Eschrig, J. Kopu, J. C. Cuevas, and G. Schön, Phys. Rev. Lett. 90, 137003 (2003).

${ }^{8}$ V. V. Ryazanov, V. A. Oboznov, A. Yu. Rusanov, A. V. Veretennikov, A. A. Golubov, and J. Aarts, Phys. Rev. Lett. 86, 2427 (2001).

${ }^{9}$ T. Kontos, M. Aprili, J. Lesueur, F. Genet, B. Stephanidis, and R. Boursier, Phys. Rev. Lett. 89, 137007 (2002).

${ }^{10}$ M. Weides, M. Kemmler, H. Kohlstedt, R. Waser, D. Koelle, R. Kleiner, and E. Goldobin, Phys. Rev. Lett. 97, 247001 (2006).

${ }^{11}$ Y. Blum, A. Tsukernik, M. Karpovski, and A. Palevski, Phys. Rev. Lett. 89, 187004 (2002).

${ }^{12}$ T. Yamashita, S. Takahashi, and S. Maekawa, Appl. Phys. Lett. 88, 132501 (2006).

${ }^{13}$ L. R. Tagirov, Phys. Rev. Lett. 83, 2058 (1999).

${ }^{14}$ A. I. Buzdin, A. V. Vedyaev, and N. V. Ryzhanova, Europhys. Lett. 48, 686 (1999).

${ }^{15}$ J. Y. Gu, C.-Y. You, J. S. Jiang, J. Pearson, Ya. B. Bazaliy, and S. D. Bader, Phys. Rev. Lett. 89, 267001 (2002).

${ }^{16}$ A. Potenza and C. H. Marrows, Phys. Rev. B 71, 180503(R) (2005).

${ }^{17}$ I. C. Moraru, W. P. Pratt, and Norman O. Birge, Phys. Rev. Lett. 96, 037004 (2006).

${ }^{18}$ A. Yu. Rusanov, S. Habraken, and J. Aarts, Phys. Rev. B 73, 060505(R) (2006).

${ }^{19}$ A. Singh, C. Sürgers, R. Hoffmann, H. v. Löhneysen T. V. Ashworth, and N. Pilet, Appl. Phys. Lett. 91, 152504 (2007).

${ }^{20}$ R. Steiner and P. Ziemann, Phys. Rev. B 74, 094504 (2006).

${ }^{21}$ J. Aarts, J. M. E. Geers, E. Brück, A. A. Golubov, and R. Coehoorn, Phys. Rev. B 56, 2779 (1997).

${ }^{22}$ H. K. Wong, B. Y. Jin, H. Q. Yang, J. B. Ketterson, and J. E. Hilliard, J. Low Temp. Phys. 63, 307 (1986).

${ }^{23}$ K. Westerholt, D. Sprungmann, H. Zabel, R. Brucas, B. Hjörvarsson, D. A. Tikhonov, and I. A. Garifullin, Phys. Rev. Lett.
95, 097003 (2005).

${ }^{24}$ Sangjun Oh, D. Youm, and M. R. Beasly, Appl. Phys. Lett. 71, 2376 (1997).

${ }^{25}$ P. Isberg, B. Hjörvarsson, R. Wäppling, E. B. Svedberg, and L. Hultman, Vacuum 48, 483 (1997).

${ }^{26}$ J. Nogués and Ivan K. Shuller, J. Magn. Magn. Mater. 192, 203 (1999).

${ }^{27}$ A. Stierle, A. Abromeit, N. Metoki, and H. Zabel, J. Appl. Phys. 73, 4808 (1993).

${ }^{28}$ L. G. Parratt, Phys. Rev. 95, 359 (1954).

${ }^{29}$ P. Poulopoulos, P. Isberg, W. Platow, W. Wisny, M. Farle, B. Hjörvarsson, and K. Baberschke, J. Magn. Magn. Mater. 170, 57 (1997).

${ }^{30}$ M. M. Schwickert, R. Coehoorn, M. A. Tomaz, E. Mayo, D. Lederman, W. L. O’Brien, Tao Lin, and G. R. Harp, Phys. Rev. B 57, 13681 (1998).

${ }^{31}$ P. Granberg, P. Isberg, E. B. Svedberg, B. Hjörvarsson, P. Nordblad, and R. Wäppling, J. Magn. Magn. Mater. 186, 154 (1998).

${ }^{32}$ T. Burkert, P. Svedlindh, G. Andersson, and B. Hjörvarsson, Phys. Rev. B 66, 220402 (2002).

${ }^{33}$ A. Broddefalk, R. Mathieu, P. Nordblad, P. Blomqvist, R. Wäppling, J. Lu, and E. Olsson, Phys. Rev. B 65, 214430 (2002).

${ }^{34}$ V. P. Romanov, S. V. Ulyanov, V. M. Uzdin, G. Nowak, M. Valada, and H. Zabel, J. Phys. D 41, 115401 (2008).

${ }^{35}$ P. F. Miceli, D. A. Neuman, and H. Zabel, Appl. Phys. Lett. 48, 24 (1986)

${ }^{36}$ W. Donner, N. Metoki, A. Abromeit, and H. Zabel, Phys. Rev. B 48, 14745 (1993).

${ }^{37}$ L. A. Patterson, Phys. Rev. 56, 978 (1939).

${ }^{38}$ R. Feidenhans'l, Surf. Sci. Rep. 10, 105 (1989).

${ }^{39}$ P. Bödeker, A. Abromeit, K. Bröhl, P. Sonntag, N. Metoki, and H. Zabel, Phys. Rev. B 47, 2353 (1993).

${ }^{40}$ B. Hjörvarsson, J. A. Dura, P. Isberg, T. U. Watanabe, T. J. Udovic, G. Andersson, and C. F. Majkrzak, Phys. Rev. Lett. 79, 901 (1997).

${ }^{41}$ I. A. Garifullin, D. A. Tikhonov, N. N. Garif'yanov, L. Lazar, Yu. V. Goryunov, S. Ya. Khlebnikov, L. R. Tagirov, K. Westerholt, and H. Zabel, Phys. Rev. B 66, 020505 (2002).

${ }^{42}$ S. Mirbt, I. A. Abrikosov, H. L. Skriver, and B. Johansson, Phys. Rev. B 55, 67 (1997).

${ }^{43}$ D. Labergerie, K. Westerholt, H. Zabel, and B. Hjörvarrsson, J. Magn. Magn. Mater. 225, 373 (2001).

${ }^{44}$ P. Granberg, P. Nordblad, P. Isberg, B. Hjörvarsson, and R. Wäppling, Phys. Rev. B 54, 1199 (1996).

${ }^{45}$ Ray Radebaugh and P. H. Keesom, Phys. Rev. 149, 217 (1966).

${ }^{46}$ S. T. Sekula and R. H. Kernohan, Phys. Rev. B 5, 904 (1972). 
${ }^{47}$ P. Koorevaar, Y. Suzuki, R. Coehoorn, and J. Aarts, Phys. Rev. B 49, 441 (1994).

${ }^{48}$ V. Leiner, K. Westerholt, A.-M. Blixt, H. Zabel, and B. Hjörvarsson, Phys. Rev. Lett. 91, 037202 (2003).

${ }^{49}$ R. A. Klemm, A. Luther, and M. R. Beasley, Phys. Rev. B 12, 877 (1975).

${ }^{50}$ D. Labergerie, C. Sutter, H. Zabel, and B. Hjörvarrsson, J. Magn. Magn. Mater. 192, 238 (1999).
${ }^{51}$ Florin Radu, Vincent Leiner, Kurt Westerholt, Hartmut Zabel, Jeffery McCord, Alexei Vorobiev, Janos Major, David Jullien, Hubert Humblot, and Francis Tasset, J. Phys.: Condens. Matter 17, 1711 (2005).

${ }^{52}$ B. Skubic, E. Holmström, D. luşan, O. Bengone, O. Eriksson, R. Brucas, B. Hjörvarsson, V. Stanciu, and P. Nordblad, Phys. Rev. Lett. 96, 057205 (2006). 